\title{
1Capturing heterogeneous infectiousness in transmission dynamic 2models of tuberculosis: a compartmental modelling approach
}

3Yayehirad A Melsew ${ }^{1,2^{*}}$, Romain Ragonnet ${ }^{1}$, Allen C Cheng ${ }^{1}$, Emma S McBryde ${ }^{3}$, James M 4Trauer ${ }^{1}$

$5^{1}$ Department of Epidemiology and Preventive Medicine, School of Public Health and 6Preventive Medicine, Monash University, 553 St Kilda Road, Melbourne, VIC 3004, 7Australia

$8^{2}$ Department of Epidemiology and Biostatistics, Institute of Public Health, University of 9Gondar, Gondar, Ethiopia

$10^{3}$ Australian Institute of Tropical Health and Medicine, James Cook University, Townsville, 11QLD 4811, AUSTRALIA

12*Corresponding Author (email: 078yayu@gmail.com or yayehirad.melsew@monash.edu,

13Tel: +61403676870$)$

14Authors' email addresses

15YAM: 078yayu@gmail.com

16RR: romain.ragonnet@monash.edu

17ACC: $\underline{\text { allen.cheng@monash.edu }}$

18ESM: emma.mcbryde@jcu.edu.au

19JMT: james.trauer@monash.edu

20

21

22

23 


\section{Abstract}

25Infectiousness heterogeneity among individuals with tuberculosis (TB) is substantial and is 26likely to have a significant impact on the long-term dynamics of TB and the effectiveness of 27interventions. However, there is a gap in capturing heterogeneous infectiousness and 28evaluating its impact on the effectiveness of interventions.

29Informed by observed distribution of secondary infections, we constructed a deterministic 30model of TB transmission using ordinary differential equations. The model incorporated 31assumption of heterogeneous infectiousness with three levels of infectivity, namely non32spreaders, low-spreaders and super-spreaders. We evaluated the effectiveness of dynamic 33transmission untargeted and targeted implementation of an intervention intended to represent 34active case finding with a point-of-care diagnostic tool. The simulated intervention detected $3520 \%$ of all TB patients who would otherwise have been missed by the health system during 36their disease episode and was compared across four epidemiological scenarios.

37Our model suggested that targeting the active case finding intervention towards super38spreaders was more effective than untargeted intervention in all setting scenarios, with more 39effectiveness in settings with low case detection and high transmission intensity. For instance, 40a targeted intervention achieved a $42.2 \%$ reduction in TB incidence, while the untargeted 41 intervention achieved only a $20.7 \%$ reduction over 20 years, given the same number of 42people treated. Although the most marked impact on equilibrium TB incidence came from the 43rate of late reactivation, the proportion of super-spreaders and their relative infectiousness 44had shown substantial impact.

45Targeting active case-finding interventions to highly infectious cases likely to be particularly 46beneficial in settings where case detection is poor. Heterogeneity-related parameters had an 
47equivalent effect to several other parameters that have been established as being very 48important to TB transmission dynamics.

49Keywords: heterogeneous infectiousness; M. tuberculosis transmission; mathematical model;

\section{Introduction}

51Tuberculosis (TB) is the world's leading cause of death from a single infectious agent, in 522019, ranking above HIV/AIDS with an estimated 10.0 million cases and 1.2 million deaths 53worldwide [1]. The World Health Organization developed the new End TB Strategy for post542015 TB elimination activities, with the ambition of a 95\% reduction in TB deaths and a 90\% 55reduction in TB incidence by 2035 by comparison to 2015 rates [2]. However, the natural 56history of TB remains poorly understood, and consequently, the uncertain potential impact of 57control interventions limits confidence about the possibility of its elimination. The 58heterogeneous transmission of Mycobacterium tuberculosis (Mtb) within populations is well59established, but its epidemiological impact is poorly understood. Moreover, up to a third of $60 \mathrm{~TB}$ cases are not diagnosed, so finding and treating infectious cases is key to achieving TB 61control, and finding and treating highly infectious people is the key to TB control in high 62transmission settings $[3,4]$.

63We define infectiousness heterogeneity as the variability in the capacity of infectious patients 64to produce secondary infections, which may be attributable to characteristics of the host, the 65agent and the environment [5-9]. Heterogeneity of infectious individuals in spreading $M t b$ 66infection is well-recognised, with a small group of highly infectious individuals producing a 67large proportion of secondary infections, while many others produce very few or none [10]. 68Previous TB genomic epidemiology has identified TB super-spreading events by quantifying 69heterogeneity in the infectiousness of TB patients through fitting a standard statistical 70distribution (the negative binomial distribution, NBD) to the distribution of secondary cases 
71produced by each infectious patient (the "offspring distribution") [11]. More recently, data

72 from TB contacts in a low-transmission setting have allowed estimation of the proportion of

73all TB patients that can be categorised as super-spreaders, finding it to be approximately $10 \%$

74[9]. In such studies, super-spreaders were typically defined as patients who produced a

75number of secondary "infections/cases" greater than the $99^{\text {th }}$ centile of a standard Poisson

76offspring distribution, with distribution mean equal to the average number of secondary

77infections per index $[9,12]$. This heterogeneity is likely to have implications for both the

78burden of disease and the effectiveness of control interventions. Thus, we propose that it is

79necessary to capture this heterogeneity when modelling TB transmission dynamics.

80Some past compartmental models of TB transmission dynamics have attempted to capture

81heterogeneity in patients' infectiousness by stratifying the active TB compartment into

82different levels of infectivity. Amongst the most typical approaches is stratification as either

83infectious (usually representing pulmonary TB) or non-infectious (extrapulmonary TB) [13-

8417]. This approach implies that all pulmonary TB patients are equally infectious, while all

85extrapulmonary patients are entirely non-infectious, and so does not capture the heterogeneity

86among pulmonary patients. Other TB models have considered sputum smear status as a factor

87in stratifying patients' levels of infectiousness, considering both smear-negative and smear-

88positive pulmonary TB to be infectious, with the relative infectiousness of smear-negative

89patients compared to smear-positive typically set between 15 and 25\% [18-33]. While

90stratifying pulmonary TB patients based on smear status captures an additional clinical

91attribute that is important in determining infectiousness, even these models still do not

92capture the full picture of TB patients' infectiousness variation, since several behavioural and

93demographic factors other than smear status can affect the level of infectiousness [5].

94An individual-based model simulated patterns of meetings and $M t b$ transmission between

95three different types of contact to determine the effects of variation in infectiousness and 
96susceptibility on transmission location. The study defined super-spreading defined based on 97the number of secondary cases instead of secondary infections and suggested that the 98majority of disease resulted from infection by a small proportion of people with TB or super99spreaders [34]. However, as TB disease activation may take long time and also depend on 100mainly on the characteristics of contact person, the definition of super-spreading used in the 101study may not show true heterogeneity in $M t b$ transmission [35]. In the current study, we 102present a deterministic compartmental $M t b$ transmission model that incorporates three levels 103of TB patients' infectivity, namely non-spreaders, low-spreaders and super-spreaders. Using 104this framework, we analysed how infectiousness heterogeneity affects disease dynamics and 105evaluated the effectiveness of a hypothetical active case finding intervention and the impact 106of targeting super-spreaders.

\section{Methods}

108Literature review

109Before constructing our model, we systematically reviewed how previous TB models have 110approached heterogeneous infectiousness [36]. In the review, we found that TB models 111frequently stratified the active TB compartment according to one or more patient-related 112 factors. We constructed a flexible model with three levels of infectiousness, without 113restricting to a given factor, and the ability to transition between these levels using empirical 114measures of heterogeneous infectiousness to parametrise the model.

\section{Empiric data}

116Data from the VTP which are stored by the Victorian Department of Health and Human 117Services (DHHS) were used for the following analysis. Index patients were classified as 118confirmed cases of TB notified from 1 January 2005 to 31 December 2015 in residents of 119Victoria. The data set includes contact tracing information and results of testing for $M t b$ 
120infection, with cases of subsequent active TB disease linked to these contact episodes now 121extending to March 2017 (see [37] for earlier publication of linkage process). We constructed 122empirical offspring distributions from the detailed contact tracing data set of the VTP.

123Ethical approval was obtained from Monash University, Human Research Ethics Committee 124(Project Number: 7776) and permission was given by the VTP and DHHS.

\section{Model structure}

126Using ordinary differential equations (ODE), we constructed a deterministic model of $M t b$ 127transmission in a hypothetical high TB burden setting. To represent heterogeneous 128infectiousness and super-spreading in $M t b$ transmission, we stratified the active TB 129compartment into three sub-classes with different levels of infectiousness: non-spreaders $\left(I_{0}\right)$, 130low-spreaders $\left(I_{1}\right)$ and super-spreaders $\left(I_{2}\right)$. We did not use any specific factor to discriminate $131 I_{0}, I_{1}$ and $I_{2}$ a priori. Instead, we use the offspring data (number of secondary infections per 132index) and a Bayesian approach to find the most realistic parameterisation to implement 133heterogeneous infectiousness in our model.

134 Fig 1 presents the model structure. The model simulates a closed population, such that births 135( $\pi)$ accrue into the fully susceptible compartment $(S)$ to replace all deaths. Natural death 136occurs from all compartments at a constant rate $(\mu)$. Susceptible individuals are dynamically 137infected, with the force of infection $(\lambda)$ contributed by super-spreaders and low-spreaders. 138Following infection, individuals enter a rapid-sojourn early latent compartment $\left(L_{A}\right)$, from 139which they may progress to one of the three active TB compartments $\left(I_{0}, I_{1}\right.$ or $\left.I_{2}\right)$ at a total 140rate of $\varepsilon$ or enter the low-risk late latent stage $\left(L_{B}\right)$ at rate $\kappa$. From the late latent state they 141may progress more slowly to disease (total rate $v$ ), also entering the active TB compartments 142i). Re-infection may occur during late latency with a reduced force of infection $\lambda_{r}\left(\lambda_{r}=r \times \lambda\right)$ 143and, if re-infection occurs, these individuals similarly enter the early latent compartment, 
144progressing to the disease at the same rates as for newly infected persons. Individuals with

145active disease may either die due to TB (rate $\mu_{i}$ ) or background mortality $(\mu)$; or

146spontaneously recover and return to the late latent state (rate $\gamma$ ); or be detected and treated by

147the health system (rate $\delta$ ) and return to the susceptible compartment $(S)$.

148Fig 1: Transmission dynamic model of TB with heterogeneous infectiousness. Boxes 149represent compartments in the model and arrows represent flows. Not shown here but 150included in the model are natural mortality from each compartment and TB-related mortality 151 from each active TB compartment $\left(I_{0}, I_{1}\right.$ and $\left.I_{2}\right)$. Red arrows represent transmission flows; 152black arrows represent disease progression processes; green arrows represent self-recovery, 153blue arrows represent detection and treatment cure.

\section{Estimation of the infectiousness parameters using empiric evidence}

155The four model parameters that capture heterogeneous infectiousness in the model were the 156proportions of non-spreaders, low-spreaders and super-spreaders, and the relative 157infectiousness of super-spreaders compared to low-spreaders. In order to estimate these 158parameters, we first determined the statistical distribution of the number of secondary 159infections per index associated with the stochastic equivalent (continuous-time Markov) 160model of our ODE-based model. We obtained a mixture of three classic statistical 161distributions: two geometric distributions for low-spreaders and super-spreaders and one 162Dirac delta distribution for non-spreaders. A detailed demonstration of this result is provided 163in the Supplementary Material.

164A Hamiltonian Monte Carlo (HMC) algorithm was used to generate 20,000 samples from the 165posterior distributions of the parameters. The 20,000 samples used for inference were 166obtained from 40,000 iterations of the HMC, burning the first 20,000 draws. Burn-in size and 167convergence were assessed by inspecting the trace plots of the estimated parameters. The 
168reported $95 \%$ credible intervals were obtained by computing the $2.5^{\text {th }}$ and $97.5^{\text {th }}$ percentiles of 169the parameters' posterior distributions. The parameter estimates from the Bayesian process is 170provided in Supplementary fig.1.

171The resulting statistical distribution was then compared in a Bayesian context with empiric 172data obtained from our previous analysis of TB contact tracing data in the Australian state of 173Victoria [9]. A comparison between the distribution of the number of secondary infections 174per index simulated by our calibrated model with the one observed empirically is presented in 175Fig 2.

176The parameter values other than those pertaining to infectiousness introduced above were 177estimated from a review of relevant evidence, with the relevant sources presented in Table $\mathbf{1 .}$

178Fig 2. Comparison of the effective modelled secondary infection distribution with empiric 179data from Victoria, Australia (red points represent the modelled distribution while blue 180points are observed empiric data).

\section{Model calibration}

182For the baseline equilibrium state, the ODE model was calibrated to a high TB burden 183settings with an incidence rate of 200 per 100,000 population per year by adjusting the 184transmission rate parameter for low-spreaders.

185The model was programmed in R 3.4.0 (R Project for Statistical Computing), and we used a 186package called 'deSolve', solver for initial value problems of ODE [38, 39]. The Bayesian 187parameter estimation was done using the package 'rstan' (v.2.18.2) [40] and the source code 188for the model is publicly available from https://github.com/Yayehirad/TB heterogeneity 
191Table 1: Model parameter values

\begin{tabular}{|c|c|c|c|c|}
\hline Corresponding model parameter & Symbol & $\begin{array}{l}\text { Base model } \\
\text { value }\end{array}$ & Uncertainty range & Source \\
\hline Natural mortality rate, per year & $\mu$ & 0.0154 & $0.0133-0.0182$ & {$[41]$} \\
\hline Rate of early progression, per year & $\varepsilon$ & 0.401 & $0.307-0.548$ & {$[42]$} \\
\hline $\begin{array}{l}\text { Rate of progression to late latency } \\
\text { after infection, per year }\end{array}$ & $\kappa$ & 3.599 & $3.452-3.693$ & {$[42]$} \\
\hline Rate of late progression, per year & $v$ & 0.002 & $0.0009-0.004$ & {$[42]$} \\
\hline $\begin{array}{l}\text { Average duration of infectiousness, } \\
\text { years }\end{array}$ & $\mathrm{D}$ & 3 & $2-4$ & {$[43]$} \\
\hline $\begin{array}{l}\text { TB-related mortality among Super- } \\
\text { spreaders, proportion }\end{array}$ & $\mu_{12}$ & 0.534 & $0.327-0.78$ & {$[43,44]$} \\
\hline $\begin{array}{l}\text { TB-case fatality among low- } \\
\text { spreaders, proportion }\end{array}$ & $\mu_{11}$ & 0.077 & $0.077-0.096$ & \\
\hline $\begin{array}{l}\text { TB-case fatality among non- } \\
\text { spreaders, proportion }\end{array}$ & $\mu_{10}$ & 0.077 & $0.077-0.096$ & \\
\hline $\begin{array}{l}\text { Conversion from non-infectious to } \\
\text { low-spreaders, proportion }\end{array}$ & $\mathrm{h}$ & 0.014 & $0-0.06$ & {$[32,45]$} \\
\hline $\begin{array}{l}\text { Conversion from low-spreaders to } \\
\text { super-spreaders, proportion }\end{array}$ & $\mathrm{j}$ & 0.014 & $0-0.06$ & Assumed \\
\hline $\begin{array}{l}\text { Relative reduction in force of re- } \\
\text { infection compared to new infection }\end{array}$ & $\mathrm{r}$ & 0.21 & $0.15-0.4$ & {$[46]$} \\
\hline \multicolumn{5}{|l|}{ Parameters calibrated from data } \\
\hline $\begin{array}{l}\text { Proportion of infectious among all } \\
\text { TB }\end{array}$ & & 0.42 & $0.39-0.46$ & $\begin{array}{l}\text { Estimated } \\
\text { from data [9] }\end{array}$ \\
\hline $\begin{array}{l}\text { Proportion of in super-spreaders } \\
\text { among infectious }\end{array}$ & & 0.18 & $0.1-0.29$ & $\begin{array}{l}\text { Estimated } \\
\text { from data [9] }\end{array}$ \\
\hline $\begin{array}{l}\text { Transmission rate per low-spreader } \\
\text { per-year }\end{array}$ & $\beta 1$ & 12 & $10-15$ & Calibrated \\
\hline $\begin{array}{l}\text { Relative infectiousness of super- } \\
\text { spreaders compared to low- } \\
\text { spreaders }\end{array}$ & $\mathrm{m}$ & 4.67 & $3.69-5.88$ & $\begin{array}{l}\text { Estimated } \\
\text { from data [9] }\end{array}$ \\
\hline \multicolumn{5}{|l|}{ Intervention parameters } \\
\hline $\begin{array}{l}\text { Baseline case detection rate, } \\
\text { proportion }\end{array}$ & CDR & 0.65 & $0.5-0.75$ & Assumed \\
\hline $\begin{array}{l}\text { Intervention (proportion of missed } \\
\text { cases detected) }\end{array}$ & $q$ & 0.2 & $0-0.4$ & Assumed \\
\hline $\begin{array}{l}\text { Proportion of intervention targeted } \\
\text { to super-spreaders }\end{array}$ & $\mathrm{d}$ & 0.5 & $0-1.0$ & Assumed \\
\hline
\end{tabular}

192

193Sensitivity analysis

194We first performed a one-way sensitivity analysis on each parameter to understand the

195sensitivity of equilibrium incidence to parameter ranges in the absence of interventions. We 196then undertook multidimensional sensitivity analyses to assess the correlation between 197equilibrium incidence and each parameter by taking 1000 samples from each parameter 198ranges with Latin hypercube sampling (LHS) technique [47, 48]. 


\section{Intervention simulations}

200We simulated an intervention intended to represent active case finding with a point-of-care 201diagnostic tool, accelerating successful diagnosis and treatment of individuals as early as 202possible before they start transmitting the infection. This could be conceptually considered as 203implemented by a highly sensitive point-of-care diagnostic technology following community 204awareness campaigns that target individuals with noticeable symptoms in order to increase 205their rate of presentation [49]. To target the intervention, clinical symptoms and behavioural 206characteristics can be used to identify those individuals who are more likely to be super207spreaders.

208We implemented an intervention capable of finding $q$ proportion of TB cases that would 209otherwise remain undetected by the passive case detection process:

210 i.e. $C D R_{\text {intervention }}=q \times\left(1-C D R_{\text {baseline }}\right)+C D R_{\text {baseline }}$, where, $C D R$ is the case detection rate, 211being the proportion of all cases detected during their disease episode and $q$ is the proportion 212of cases detected under the intervention scenario that would otherwise be missed by the 213baseline CDR. We set the maximum attainable level of CDR in all intervention scenarios 214including targeting super-spreaders at 90\%. In targeting the intervention towards super215spreaders, the maximum possible CDR targeted to super-spreaders under the intervention 216occurs when the CDR for non-spreaders and low-spreaders is kept at the baseline. With this 217restriction, we defined a single parameter $(d)$ to represent the extent of intervention targeting 218relative to the maximum amount of targeting possible, which ranges from zero to one, with 219zero representing untargeted intervention and one representing $100 \%$ targeting. That is, the 220CDR targeted to super-spreaders under the intervention scenario was $C D R_{\text {intervention }}$ plus $d$ 221multiplied by the difference between the maximum possible CDR and $C D R_{\text {intervention. }}$ This 
222implementation ensures equivalent effort or the number of people treated for untargeted and 223targeted interventions; details of these calculations are given in the Supplementary Material.

224We then evaluated the effectiveness of targeting super-spreaders $(d>0)$ compared to 225untargeted intervention $(d=0)$ in four independent scenarios. These four scenarios were 226settings with high transmission and high case detection, high transmission and low case 227detection, low transmission and high case detection, and low transmission and low case 228detection. These four scenarios were simulated by calibrating the transmission parameter for 229low-spreaders and CDR. In addition, we evaluated the impact of levels of targeting $(d)$ for 230different coverage levels of the intervention $(q)$ in these four setting scenarios, while other 231model parameters were sampled from the same plausible ranges as in the baseline sensitivity 232analysis using LHS.

\section{Results}

\section{Baseline sensitivity analyses}

235The sensitivity analyses show that the most marked impact on equilibrium incidence arose 236from the rate of late TB progression followed by the CDR (Fig 3). The proportion of super237spreaders among persons with infectious TB and the relative infectiousness of super238spreaders compared to low-spreaders also had a substantial impact. Supplementary Fig 2 239presents a multidimensional sensitivity analysis, using the LHS method to sample 1000 240parameter sets, which was consistent with the results of the one-way sensitivity analysis.

241Fig 3: One-way sensitivity analyses of equilibrium TB incidence to variation between 242extremes of plausible parameter values. Maximum and minimum parameter values are given 243in Table 1. Values on the $x$-axis represent the equilibrium TB incidence, and the vertical line 244indicates the equilibrium incidence obtained with the baseline parameter set. 


\section{Impact of targeted interventions}

246Targeting the active case finding intervention towards super-spreaders was more effective 247than mass intervention in all setting scenarios. However, its effectiveness was particularly 248marked in settings with low case detection, regardless of transmission rates. For example, in 249the first scenario of both high transmission and high CDR, a 20\% untargeted active case 250 finding intervention led to a $22.8 \%$ reduction in incidence over 20 years, while with an $80 \%$ 251 targeting an equal active case finding intervention resulted in a $35.9 \%$ reduction in $\mathrm{TB}$ 252incidence over the same period. However, in the scenario of high transmission but low CDR, 253the targeted intervention is much more effective (achieving a $42.2 \%$ reduction) than the 254untargeted intervention, which only reduced incidence by $22.8 \%$ over 20 years, considering a $25520 \%$ active case finding and an $80 \%$ level of targeting. Interventions in settings of low 256transmission and high case detection rates had a relatively minor impact on the burden 257(Supplementary Fig. 3).

258We evaluated the impact of intensifying active case finding $(q)$ from zero to $40 \%$ detection 259and varying the proportion of this intervention targeting the super-spreaders $(d)$. As shown in 260Fig 4, both these quantities are essential - i.e. the increase in CDR and the extent of targeting 261provided the increase in CDR is more than negligible. The most significant impact of 262targeting was in the setting of high transmission and low CDR at all coverage levels of the 263intervention. Targeting super-spreaders was more effective at all levels of relative CDR 264increases in settings with high transmission and low case detection (Panel B), while in 265settings with high transmission and high case detection, targeting super-spreaders has a 266significant effect if the relative increase in CDR is low (Panel A). 
269Fig 4: Comparison of extent of active case finding and the proportion of targeting to super-

270spreaders on the 20-year projected relative reduction in TB incidence under the four

271scenarios. A) High transmission and high case detection setting; B) high transmission and

272low case detection; C) low transmission and high case detection; D) low transmission and

273low case detection.

274Fig 5 presents the results of the one-way sensitivity analysis performed to observe the impact 275of high and low extremes of parameter values on the intervention's 20 -year projected relative 276incidence reduction. The sensitivity analyses show that the most marked impact on 277intervention effectiveness arose from the rate of late progression, followed by the rate of 278early progression and the proportion of super-spreaders. Notably, while higher rates of late 279progression were correlated with lower effectiveness of the active case finding intervention, 280higher rates of early progression resulted in greater reductions in the incidence.

281Supplementary fig 4 presents the results of the multidimensional sensitivity analyses 282assessing the impact of parameter ranges on intervention effectiveness (relative reduction in 283annual incidence with 20 -years projections). This multidimensional analysis supports the 284results of the one-way sensitivity analysis: that the proportion of super-spreaders had a 285considerable impact on intervention effectiveness, and was the most important parameter 286after the disease progression parameters.

287Fig 5: One-way sensitivity analyses of 20-year projected relative reduction in incidence to 288 variation between extremes of plausible parameter values. The maximum and minimum 289parameter values are given in Table 1. Values on the $x$-axis represent the relative reduction 290 in incidence following the implementation of a $20 \%$ active case finding intervention with $29180 \%$ targeting. The vertical line indicates the relative reduction in incidence obtained with 292the baseline parameter set. 


\section{Discussion}

294Our model suggested that targeted active case finding interventions directed toward people 295likely to be super-spreaders could have a substantial impact on the TB burden, particularly in 296settings with high transmission but low case detection rates. This model also showed that 297parameters related to infectiousness heterogeneity such as the proportion of super-spreaders 298and their relative infectiousness compared to other low-spreaders were as crucial as other 299previously recognised epidemiological parameters, such as the latency progression 300parameters, in determining the burden of TB. The current model is also able to incorporate a 301range of commonly used approaches to capturing heterogeneous infectiousness of active TB 302cases. The choice of three infectiousness levels for active TB and the values assigned to each 303compartment was based on empirical data estimates, strengthening the validity of our 304findings.

305Active case finding has been described as "turning off the tap" in TB control intervention 306strategies since it represents a method of identifying individuals with TB and promptly 307initiating treatment to avoid further onward transmissions [50]. Previous TB modelling has 308suggested that active case finding with highly sensitive diagnostic tools can reduce delay to 309treatment and so significantly reduce TB transmission [51]. The use of point-of-care 310diagnostic technologies with high sensitivity to detect persons with TB soon after they begin 311to transmit, such as GeneXpert Omni [49], could enable the implementation of active case 312 finding interventions similar to that considered in this study. In fact, any TB active case 313finding intervention necessarily incorporates some level of targeting towards the highly 314infectious individuals, because highly infectious patients' disease characteristics (such as 315higher sputum-bacilli concentration and lung-cavitation) make them more easily detectable 316with existing microbiological and radiological diagnostic tools. This means that 317implementing more sensitive diagnostics may paradoxically decrease targeting of super- 
318spreaders - whereas traditional smear-based interventions might ensure that resources are 319targeted to the most infectious.. In agreement with previous modelling [52], our model 320suggested that active case finding interventions are particularly valuable in high transmission 321and low case detection settings to limit onward transmission. In the implementation of the 322intervention, although we did not perform cost-effectiveness analyses, our study shows that 323finding super-spreaders (less than $10 \%$ of all TB cases) is equally as effective 324epidemiologically as finding $40 \%$ of general TB population. In addition, the cost of 325identifying and treating super-spreaders that only comprises less than $10 \%$ of total TB cases 326can be compared with the cost of treating all active TB cases in a population. Our findings 327concerning the effectiveness of interventions can provide broad directions to future TB 328modellers and policymakers, although predictions from our model are not intended to provide 329location-specific estimates.

330Our sensitivity analyses showed that a higher late reactivation rate had a negative impact on 331the effectiveness of active case finding interventions, while higher rates of early progression 332 increased the impact on the 20 -year projected reduction of incidence following the 333intervention. This suggests that active case finding interventions are more effective if the TB 334epidemic is more dependent on early progression and intense recent transmission than late 335TB reactivation since the intervention would have a more significant effect on rapidly 336reducing transmission. A recent study showed that variation in the latency progression 337parameters had important impacts on model predictions around the effectiveness of 338preventive therapy interventions [53], while our analysis complements this finding by 339showing that these parameter variations also have indispensable effects on predictions 340regarding the effectiveness of case-finding interventions.

341In our analyses, the rate of conversion from low-spreader to super-spreader had little 342significance, and impact of the rate of conversion from non-infectious to low-spreaders was 
343negligible. It is known that active TB cases' ability to spread Mtb may increase as they 344progress clinically to more severe disease, e.g. from smear-negative to smear-positive or from 345non-cavitary to cavitary-TB [54]. In previous TB models, spontaneous conversion from less 346infectious to more infectious states such as smear-negative to smear-positive was included 347 with rates around $1.5 \%$ per year $[18,25,32,45,55-57]$. These parameters do not affect model 348outputs substantially, such that they can be omitted for model simplicity if the actual values 349are consistent with what has previously been assumed. Nevertheless, the exact values of these 350quantities remain uncertain, and future research to refine these quantities may modify this 351conclusion.

352The current model structure enhances flexibility around the assumption of infectiousness 353heterogeneity that allowed for reflection of a broad range of factors that can alter active TB 354 cases infectivity level $[5,58]$. Thus, the model is able to incorporate many previous 355compartmental modelling structural approaches, including those that stratified active TB 356cases' infectivity into two levels, as non-infectious and infectious [13-17], or model 357structures that incorporate three levels of infectivity, as non-infectious, smear-negative 358infectious and smear-positive infectious $[30,31,59]$. However, in the application of this 359model to a particular epidemiological setting, data to estimate the proportion of super360 spreaders is essential, since this information has a particularly marked impact on both 361baseline disease burden and intervention effectiveness.

$362 \mathrm{We}$ used empirical data to parameterise our model, but a noteworthy limitation is that these 363results may not be generalizable to other settings. However, we are not aware of any past 364work that has used empiric data to inform model parameters on the proportions of infectious 365TB and super-spreaders at all. As with any model-based analyses, our study has limitations 366that arise from its assumptions. In addition to those introduced above, our model is not 367intended to represent a specific setting, and as such does not incorporate stratification by age, 
368HIV or multidrug-resistant TB. The other limitation is that we used a very simplified 369implementation of an active case finding intervention with a theoretical point-of-care 370diagnostic tool, without considering all the complexities in TB diagnosis and treatment 371implementation.

\section{Conclusions}

373The approaches we used to inform model parameters related to infectiousness heterogeneity 374 from the observed distribution of secondary infections can be useful for future modelling 375studies of TB, in particular, and other infectious diseases, in general. The principles of 376implementation could be used in future TB modelling studies that represent specific 377epidemiological settings, especially when TB contact investigation data are available to 378estimate the proportion of super-spreaders. In the usage of advanced point-of-care 379technologies, targeting active case-finding interventions to super-spreaders is likely to be 380especially beneficial in low CDR settings.

\section{Acknowledgements}

382The authors would like to thank Monash University for providing the PhD scholarship to 383YAM.

\section{Funding}

385This research did not receive any specific grant from funding agencies in the public, 386 commercial, or not-for-profit sectors.

\section{Availability of data and materials}

388The R-code used to analyse this model is publicly available.

\section{Authors' contributions}


bioRxiv preprint doi: https:/doi.org/10.1101/2020.06.26.173104: this version posted June 26, 2020. The copyright holder for this preprint (which was not certified by peer review) is the author/funder, who has granted bioRxiv a license to display the preprint in perpetuity. It is made available under aCC-BY 4.0 International license.

390YAM, RR and JMT conceived the study. YAM developed structure and ODE of the model, 391which was reviewed by JMT and RR. ESM, ACA, JMT conceived the strategy of the 392intervention in the model. YAM coded the model in R that RR and JMT reviewed and 393evaluated. YAM drafted the manuscript, and all authors provided input into revisions and 394approved the final draft for submission.

\section{Ethics approval and consent to participate}

396Not applicable

\section{Consent for publication}

398Not applicable

\section{Competing interests}

400The authors declare that they have no competing interests.

\section{Supplementary material}

402Supplementary Material. doc

\section{References}

4041. WHO. Global Tuberculosis Report 2019. Geneva: 2019.

4052. WHO. The End TB Strategy: Global strategy and targets for tuberculosis prevention, 406care and control after 2015. Geneva, Switzerland 2014.

4073. Herbert N, George A, Sharma V, Oliver M, Oxley A, Raviglione M, et al. World TB 408Day 2014: finding the missing 3 million. The Lancet. 2014;383(9922):1016-8.

4094. Zumla A, George A, Sharma V, Herbert RHN, Oxley A, Oliver M. The WHO 2014 410global tuberculosis report - further to go. The Lancet Global Health. 2015;3(1):e10-e2.

4115. Melsew Y, Doan T, Gambhir M, Cheng A, McBryde E, Trauer J. Risk factors for 412infectiousness of patients with tuberculosis: a systematic review and meta-analysis. 413Epidemiol Infect. 2018:1-9.

4146. Turner RD, Chiu C, Churchyard GJ, Esmail H, Lewinsohn DM, Gandhi NR, et al. 415Tuberculosis infectiousness and host susceptibility. The Journal of infectious diseases. 4162017;216(suppl_6):S636-S43.

4177. Issarow CM, Mulder N, Wood R. Environmental and social factors impacting on 418epidemic and endemic tuberculosis: a modelling analysis. Royal Society open science. 4192018;5(1):170726. 
bioRxiv preprint doi: https:/doi.org/10.1101/2020.06.26.173104; this version posted June 26, 2020. The copyright holder for this preprint (which was not certified by peer review) is the author/funder, who has granted bioRxiv a license to display the preprint in perpetuity. It is made available under aCC-BY 4.0 International license.

4208. Trauer JM, Dodd PJ, Gomes MGM, Gomez GB, Houben RMGJ, McBryde ES, et al. 421The Importance of Heterogeneity to the Epidemiology of Tuberculosis. Clin Infect Dis. 4222018;69(1):159-66. doi: 10.1093/cid/ciy938.

4239. Melsew YA, Gambhir M, Cheng AC, McBryde ES, Denholm JT, Tay EL, et al. The 424role of super-spreading events in Mycobacterium tuberculosis transmission: evidence from 425contact tracing. BMC Infect Dis. 2019;19(1):244. doi: 10.1186/s12879-019-3870-1. 42610. Lloyd-Smith JO, Schreiber SJ, Getz WM, editors. Moving beyond averages: 427Individual-level variation in. Mathematical Studies on Human Disease Dynamics: Emerging 428Paradigms and Challenges: AMS-IMS-SIAM Joint Summer Research Conference on 429Modeling the Dynamics of Human Diseases: Emerging Paradigms and Challenges, July 1743021, 2005, Snowbird, Utah; 2006: American Mathematical Soc.

43111. Ypma RJ, Altes HK, van Soolingen D, Wallinga J, van Ballegooijen WM. A sign of 432superspreading in tuberculosis: highly skewed distribution of genotypic cluster sizes. 433Epidemiology. 2013;24(3):395-400.

43412. Lloyd-Smith JO, Schreiber SJ, Kopp PE, Getz WM. Superspreading and the effect of 435individual variation on disease emergence. Nature. 2005;438(7066):355-9.

43613. Aparicio JP, Castillo-Chavez C. Mathematical modelling of tuberculosis epidemics. 437Mathematical Biosciences \& Engineering: MBE. 2009;6(2):209-37. PubMed PMID: 43819364150.

43914. Hickson R, Mercer G, Lokuge K, editors. Sensitivity analysis of a model for 440tuberculosis. 19th international congress on modelling and simulation; 2011.

44115. Hickson RI, Mercer GN, Lokuge KM. A metapopulation model of tuberculosis 442transmission with a case study from high to low burden areas. PLoS One. 2012;7(4):e34411. 44316. Korthals Altes H, Kloet S, Cobelens F, Bootsma M. Latent tuberculosis infection in 444foreign-born communities: Import vs. transmission in The Netherlands derived through 445mathematical modelling.[Erratum appears in PLoS One. 2018 May 24;13(5):e0198376; 446PMID: 29795693]. PLoS ONE [Electronic Resource]. 2018;13(2):e0192282. PubMed PMID: 44729444122.

44817. Thomas EG, Barrington HE, Lokuge KM, Mercer GN. Modelling the spread of 449tuberculosis, including drug resistance and HIV: a case study in Papua New Guinea's 450Western Province. The ANZIAM Journal. 2010;52(1):26-45.

45118. Dowdy DW, Chaisson RE, Maartens G, Corbett EL, Dorman SE. Impact of enhanced 452tuberculosis diagnosis in South Africa: a mathematical model of expanded culture and drug 453susceptibility testing. Proceedings of the National Academy of Sciences. 2008.

45419. Dowdy DW, Chaisson RE, Moulton LH, Dorman SE. The potential impact of 455enhanced diagnostic techniques for tuberculosis driven by HIV: a mathematical model. 456AIDS. 2006;20(5):751-62. PubMed PMID: 16514306.

45720. Dowdy DW, Davis JL, den Boon S, Walter ND, Katamba A, Cattamanchi A. 458Population-level impact of same-day microscopy and Xpert MTB/RIF for tuberculosis 459diagnosis in Africa. PLoS One. 2013;8(8):e70485.

46021. Lin H-H, Dowdy D, Dye C, Murray M, Cohen T. The impact of new tuberculosis 461diagnostics on transmission: why context matters. Bull World Health Organ. 2012;90:739-47. 46222. Oxlade O, Schwartzman K, Benedetti A, Pai M, Heymann J, Menzies D. Developing 463a tuberculosis transmission model that accounts for changes in population health. Med Decis 464Making. 2011;31(1):53-68.

46523. Pandey S, Chadha V, Laxminarayan R, Arinaminpathy N. Estimating tuberculosis 466incidence from primary survey data: a mathematical modeling approach. The International 467Journal of Tuberculosis and Lung Disease. 2017;21(4):366-74. 
bioRxiv preprint doi: https//doi.org/10.1101/2020.06.26.173104; this version posted June 26, 2020. The copyright holder for this preprint (which was not certified by peer review) is the author/funder, who has granted bioRxiv a license to display the preprint in perpetuity. It is made available under aCC-BY 4.0 International license.

46824. Sun AY, Pai M, Salje H, Satyanarayana S, Deo S, Dowdy DW. Modeling the impact 469of alternative strategies for rapid molecular diagnosis of tuberculosis in Southeast Asia. Am J 470Epidemiol. 2013;178(12):s1740-9.

47125. Houben R, Lalli M, Sumner T, Hamilton M, Pedrazzoli D, Bonsu F, et al. TIME 472Impact-a new user-friendly tuberculosis (TB) model to inform TB policy decisions. BMC 473Med. 2016;14(1):56.

47426. Menzies NA, Cohen T, Hill AN, Yaesoubi R, Galer K, Wolf E, et al. Prospects for 475Tuberculosis Elimination in the United States: Results of a Transmission Dynamic Model. 476Am J Epidemiol. 2018.

47727. Menzies NA, Cohen T, Lin H-H, Murray M, Salomon JA. Population health impact 478and cost-effectiveness of tuberculosis diagnosis with Xpert MTB/RIF: a dynamic simulation 479and economic evaluation. PLoS Med. 2012;9(11):e1001347.

48028. Garcia A, Maccario J, Richardson S. Modelling the annual risk of tuberculosis 481infection. Int J Epidemiol. 1997;26(1):190-203. PubMed PMID: 9126520.

48229. Dowdy DW, Andrews JR, Dodd PJ, Gilman RH. A user-friendly, open-source tool to 483project impact and cost of diagnostic tests for tuberculosis. Elife. 2014;3:e02565.

48430. Arregui S, Iglesias MJ, Samper S, Marinova D, Martin C, Sanz J, et al. Data-driven 485model for the assessment of Mycobacterium tuberculosis transmission in evolving 486demographic structures. Proc Natl Acad Sci U S A. 2018;115(14):E3238-E45. PubMed 487PMID: 29563223.

48831. Dowdy DW, Lotia I, Azman AS, Creswell J, Sahu S, Khan AJ. Population-level 489impact of active tuberculosis case finding in an Asian megacity. PLoS One. 4902013;8(10):e77517.

49132. Salomon JA, Lloyd-Smith JO, Getz WM, Resch S, Sánchez MS, Porco TC, et al. 492Prospects for advancing tuberculosis control efforts through novel therapies. PLoS Med. 4932006;3(8):e273.

49433. Vynnycky E, Sumner T, Fielding KL, Lewis JJ, Cox AP, Hayes RJ, et al. 495Tuberculosis control in South African gold mines: mathematical modeling of a trial of 496community-wide isoniazid preventive therapy. Am J Epidemiol. 2015;181(8):619-32.

49734. McCreesh N, White RG. An explanation for the low proportion of tuberculosis that 498results from transmission between household and known social contacts. Sci Rep. 4992018;8(1):5382.

50035. Melsew YA, Cheng AC, McBryde ES, Denholm JT, Tay E, Ragonnet R, et al. 501Profiles of tuberculosis disease activation among contacts of patients with tuberculosis. Eur 502Respir J. 2019;54(4):1900353.

50336. Melsew YA, Adekunle AI, Cheng AC, McBryde ES, Ragonnet R, Trauer JM. 504Heterogeneous infectiousness in mathematical models of tuberculosis: A systematic review. 505Epidemics. 2019:100374.

50637. Moyo N, Tay E, Denholm J. Evaluation of tuberculin skin testing in tuberculosis 507contacts in Victoria, Australia, 2005-2013. Public health action. 2015;5(3):188-93.

50838. RCoreTeam. R: A Language and Environment for Statistical Computing. Vienna, 509Austria: R Foundation for Statistical Computing; 2014.

51039. Soetaert K, Petzoldt T, Setzer RW. Solving differential equations in R: package 511deSolve. Journal of Statistical Software. 2010;33.

51240. Team SD. RStan: the R interface to Stan, Version 2.18.2. 2018.

51341. WHO. ETHIOPIA:FactsheetsofHealthStatistics 2016. 2016.

51442. Ragonnet R, Trauer JM, Scott N, Meehan MT, Denholm JT, McBryde ES. Optimally 515capturing latency dynamics in models of tuberculosis transmission. Epidemics. 2017. 
bioRxiv preprint doi: https://doi org/10.1101/2020.06.26.173104; this version posted June 26 , 2020. The copyright holder for this preprint (which was not certified by peer review) is the author/funder, who has granted bioRxiv a license to display the preprint in perpetuity. It is made available under aCC-BY 4.0 International license.

51643. Tiemersma EW, van der Werf MJ, Borgdorff MW, Williams BG, Nagelkerke NJ. 517Natural history of tuberculosis: duration and fatality of untreated pulmonary tuberculosis in 518HIV negative patients: a systematic review. PLoS One. 2011;6(4):e17601.

51944. Trauer JM, Denholm JT, McBryde ES. Construction of a mathematical model for 520tuberculosis transmission in highly endemic regions of the Asia-Pacific. J Theor Biol. 5212014;358:74-84.

52245. Hughes GR, Currie CS, Corbett EL, editors. Modeling tuberculosis in areas of high 523HIV prevalence. Simulation Conference, 2006 WSC 06 Proceedings of the Winter; 2006: 524IEEE.

52546. Andrews JR, Noubary F, Walensky RP, Cerda R, Losina E, Horsburgh CR. Risk of 526progression to active tuberculosis following reinfection with Mycobacterium tuberculosis. 527Clin Infect Dis. 2012;54(6):784-91.

52847. Drake JM, Rohani P. Sensitivity analysis of deterministic models through Latin 529hypercube sampling: A model for the spread of Ebola virus disease2015. Available from: 530https://daphnia.ecology.uga.edu/drakelab/wp-content/uploads/2015/07/sensitivity-ebola.pdf. 53148. Gomero B. Latin Hypercube Sampling and Partial Rank Correlation Coefficient 532Analysis Applied to an Optimal Control Problem: University of Tennessee; 2012.

53349. FIND Ca. World's most portable molecular diagnostics system unveiled at AAC. 5342015 [cited 2019 May 14]. Available from:

535https://www.prnewswire.com/news-releases/worlds-most-portable-molecular-diagnostics536system-unveiled-at-aacc-300119213.html.

53750. Yuen CM, Amanullah F, Dharmadhikari A, Nardell EA, Seddon JA, Vasilyeva I, et 538al. Turning off the tap: stopping tuberculosis transmission through active case-finding and 539prompt effective treatment. The Lancet. 2015;386(10010):2334-43.

54051. Uys PW, Warren R, van Helden PD, Murray M, Victor TC. Potential of rapid 541diagnosis for controlling drug-susceptible and drug-resistant tuberculosis in communities 542where Mycobacterium tuberculosis infections are highly prevalent. J Clin Microbiol. 5432009;47(5):1484-90.

54452. Dowdy DW, Basu S, Andrews JR. Is passive diagnosis enough? The impact of 545 subclinical disease on diagnostic strategies for tuberculosis. Am J Respir Crit Care Med. 5462013;187(5):543-51.

54753. Sumner T, White RG. Modelling the impact of tuberculosis preventive therapy: the 548importance of disease progression assumptions. bioRxiv. 2019:666669.

54954. Pai M, Behr MA, Dowdy D, Dheda K, Divangahi M, Boehme CC, et al. Tuberculosis. 550Nature Reviews Disease Primers. 2016;2:16076. doi: 10.1038/nrdp.2016.76.

55155. Knight GM, Griffiths UK, Sumner T, Laurence YV, Gheorghe A, Vassall A, et al. 552Impact and cost-effectiveness of new tuberculosis vaccines in low-and middle-income 553countries. Proceedings of the National Academy of Sciences. 2014;111(43):15520-5. 55456. Legrand J, Sanchez A, Le Pont F, Camacho L, Larouze B. Modeling the impact of 555tuberculosis control strategies in highly endemic overcrowded prisons. PLoS One. 5562008;3(5):e2100.

55757. Mellor GR, Currie CS, Corbett EL. Incorporating household structure into a discrete558event simulation model of tuberculosis and HIV. ACM Transactions on Modeling and 559Computer Simulation (TOMACS). 2011;21(4):26.

56058. Grassly NC, Fraser C. Mathematical models of infectious disease transmission. 561Nature Reviews Microbiology. 2008;6(6).

56259. Abu-Raddad LJ, Sabatelli L, Achterberg JT, Sugimoto JD, Longini IM, Dye C, et al. 563Epidemiological benefits of more-effective tuberculosis vaccines, drugs, and diagnostics. 564Proceedings of the National Academy of Sciences. 2009;106(33):13980-5. 
bioRxiv preprint doi: https://doi.org/10.1101/2020.06.26.173104; this version posted June 26, 2020. The copyright holder for this preprint (which was not certified by peer review) is the author/funder, who has granted bioRxiv a license to display the preprint in perpetuity. It is made available under aCC-BY 4.0 International license.

565

566

567

568 


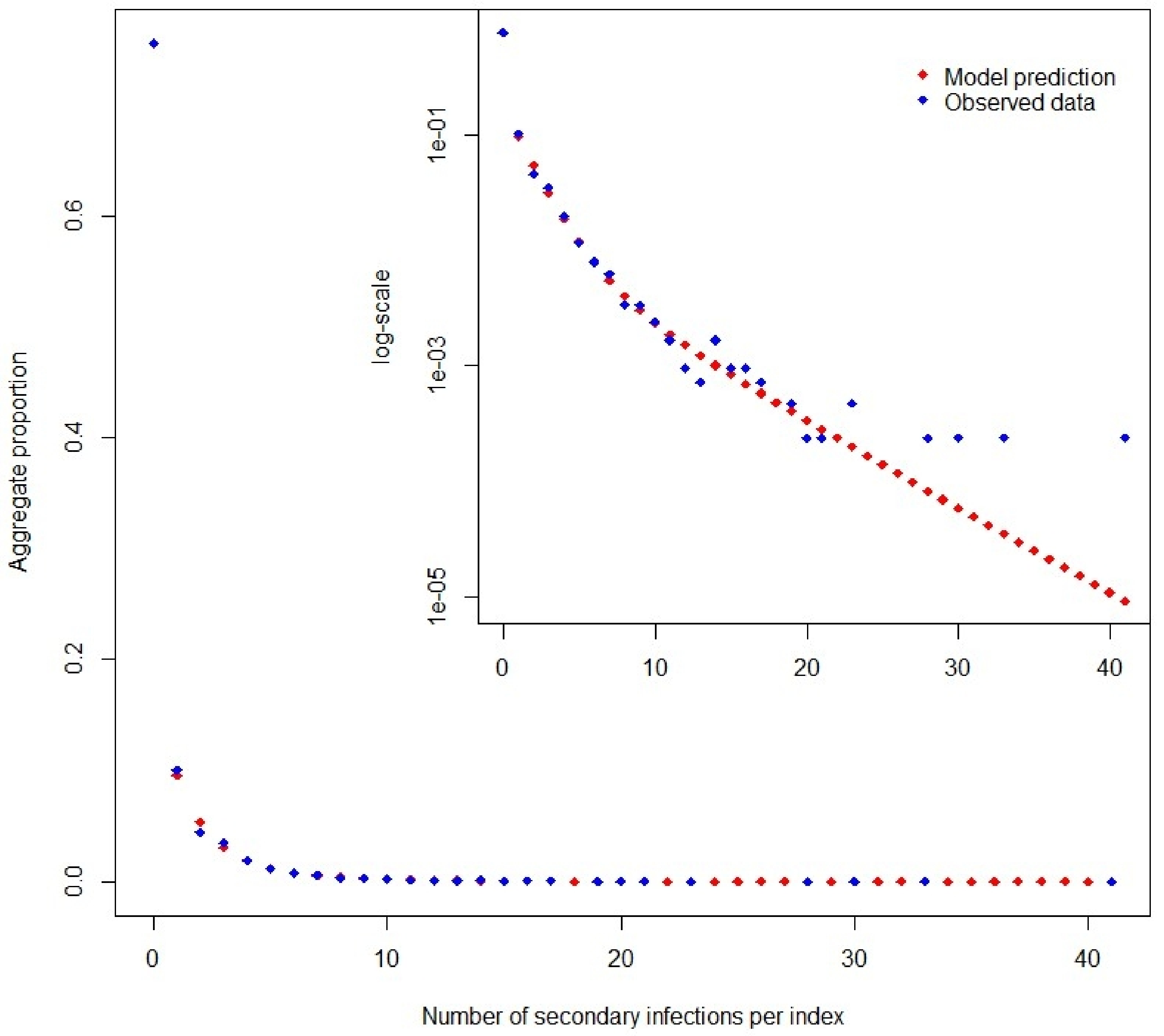

Fig 2: Comparison of the effective modelled secondary infe 
Rate of late TB progression, $v$

Case detection rate, $\mathrm{CDR}$

Rate of early TB progression, $\varepsilon$

Proportion super-spreaders, among infectious

Transmission rate per low-spreader, $\beta 1$

Relative infectiousness of super-spreaders, $m$

Proportion of infectious TB

Rate of conversion from low-spreaders to super-spreaders, $\mathrm{j}$

Natural mortality rate, $\mu$ Rate of conversion from non-infectious to low-spreaders, $h$

TB-related mortality, super-spreaders, $\mu / 2$

TB-related mortality, non-spreaders, $\mu \mathrm{IO}$

TB-related mortality, low-spreaders, $\mu \mathrm{I} 1$

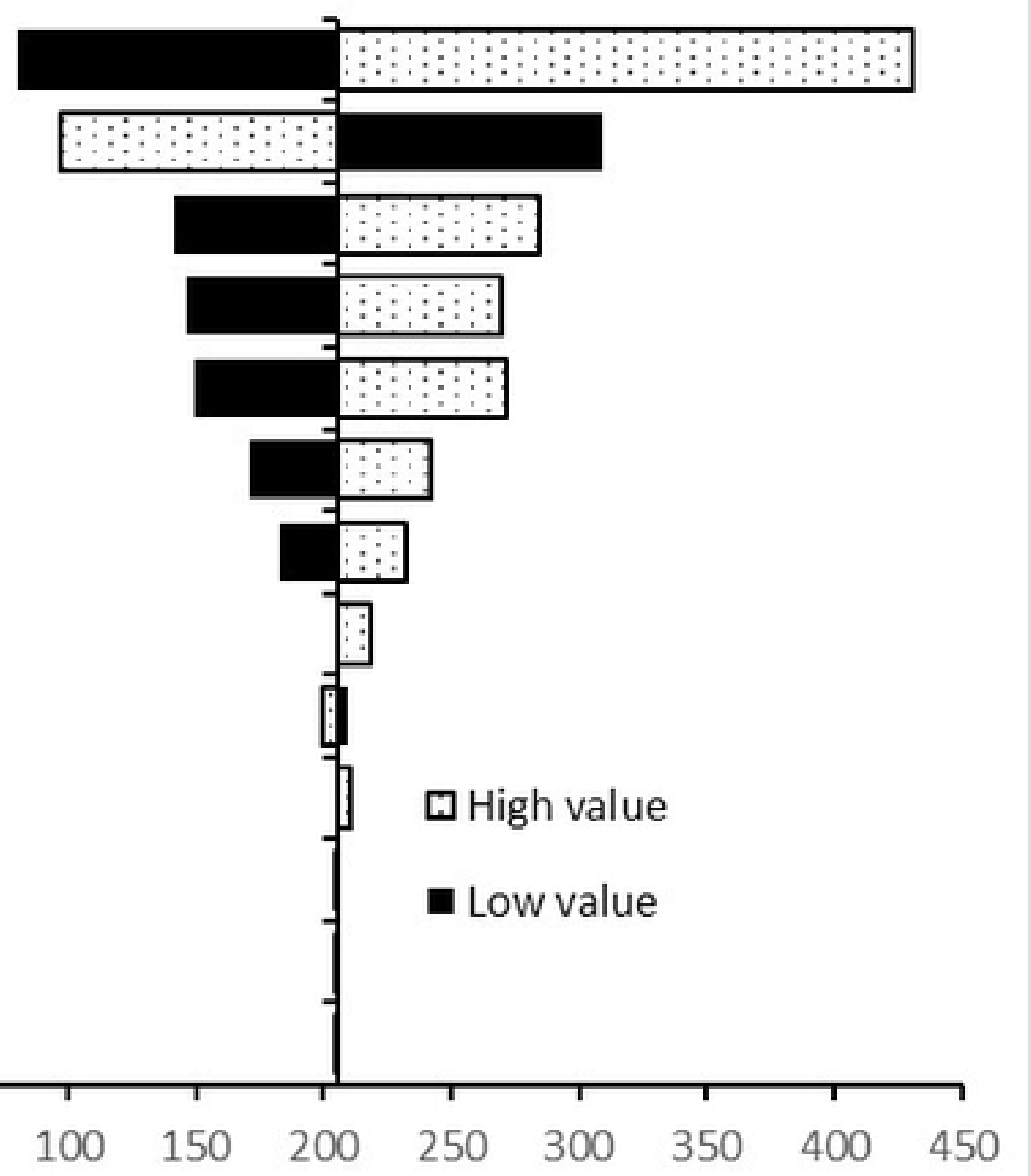

Equilibrium incidence per 100,000 population

Fig 3: One-way sensitivity analyses of equilibrium TB incidence to 
Rate of late progression per year, $v$

Rate of early progression per year, $\varepsilon$

Proportion of super-spreaders among infectious

Natural mortality rate per year, $\mu$

Case detection rate, CDR

Relative infectiousness of super-spreaders, $m$ Rate of conversion low-spreaders to super-spreaders, $\mathrm{j}$

Transmission rate per low-spreader per-year, $\beta 1$ Average duration of infectiousness, D Proportion infectious TB

TB-related mortality rate, super-spreaders, $\mu \mathrm{I} 2$

TB-case fatality among, non-spreaders $\mu \mathrm{IO}$ Rate of conversion non-infectious to low-spreaders, $\mathrm{h}$

TB-case fatality among low-spreaders, $\mu$ I1

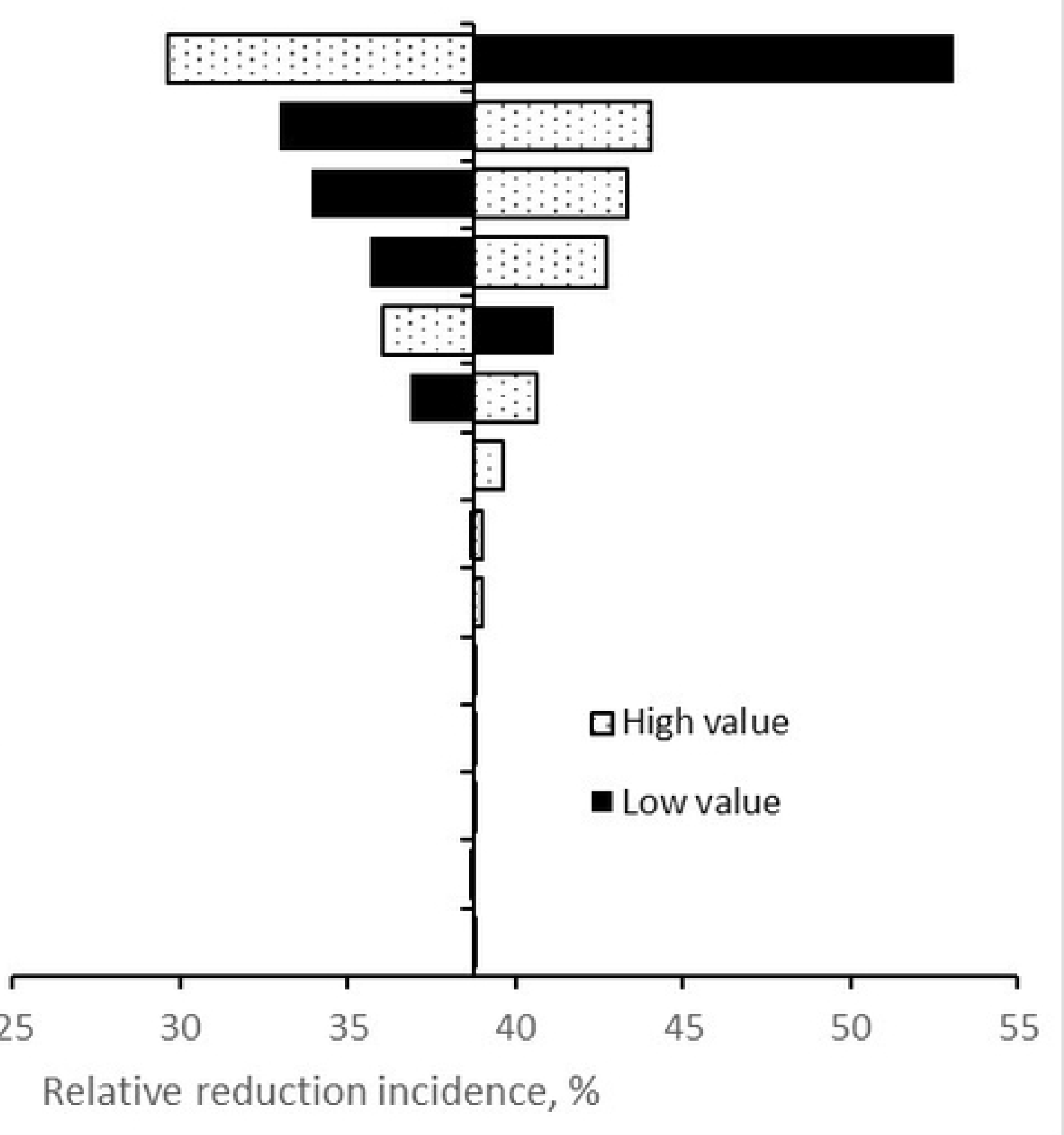

\section{Fig 5: One-way sensitivity analyses of 20 -year projected relative}

